\title{
References
}

[A1] J. Ax and S. Kochen, Diophantine problems over local fields III, Ann. of Math. (1966), 83, 437-456.

[A2] J. Ax and S. Kochen, Diophantine problems over local fields I and II, Amer. Jour. Math. (1965 and 1966), 87, 605-630 and 631-648.

[Ba1] J. T. Baldwin, Ph.D. Thesis, Simon Fraser University (1971).

[Bar1] K. T. Barwise, Infinitary logic and admissible sets, Jour. Symb. Log. (1969), 34, 226-254.

[Bl1] L. Blum, Ph.D. Thesis, Massachusetts Institute of Technology, 1968.

[Ch1] C. C. Chang and H. J. Keisler, Theory of Models, North-Holland (1990), xvi+650pp.

[E1] Y. Ershov, On the elementary theory of maximal normal fields, Algebra i Logica (1965), 31-70.

[Gö1] K. Gödel, The Consistency of the Generalized Continuum Hypothesis, Princeton University Press (1940).

[Ka1] I. Kaplansky, Maximal fields with valuations, Duke Math. Jour. (1942), 313-321.

[Ke1] H. J. Keisler, Model Theory for Infinitary Logic, North-Holland (1971).

[Ke2] H. J. Keisler, Ultraproducts and elementary classes, Indag. Math. (1961), 23, 477-495.

[Ko1] S. Kochen, Ultraproducts in the theory of models, Ann. of Math. (1962), $74,221-261$.

[La1] A. H. Lachlan, On the number of countable models of a countable superstable theory, in Logic, Methodology and Philosophy of Science IV, NorthHolland (1973), 45-56.

[La2] A. H. Lachlan, A property of stable theories, Fund. Math. (1972), 77, 9-20.

[Ma1] W. Marsh, Ph.D. Thesis, Dartmouth College (1966).

[Mo1] M. Morley, The number of countable models, Jour. Symb. Log. (1970), $35,14-18$.

[P11] R. Platek, Ph.D. Thesis, Stanford University (1966).

[Ri1] J. F. Ritt, Differential Algebra, Amer. Math. Soc. Publication (1950).

[Ro1] A. Robinson, On the concept of a differentially closed field, Bull. Res. Council of Israel (1959), 113-128. 
[Ro2] A. Robinson, Problems and methods of model theory, Centro Internazionale Matematico Estivo, Varenna (1968).

[Ros1] J. Rosenthal, A new proof of a theorem of Shelah, Jour. Symb. Log. (1972), 133-134.

[Sa1] G. E. Sacks, Effective bounds on Morley rank, Fund. Math. (1979), 103, 111-121.

[Sa2] G. E. Sacks, Bounds on weak scattering, Notre Dame Jour. of Formal Log. (2007), 48, 5-34.

[Sc1] D. Scott, Logic with denumerably long formulas and finite strings of quantifiers, The Theory of Models, North-Holland, 1965.

[Se1] A. Seidenberg, An elimination theory for differential algebra, Univ. of Calif. (1956), 31-66.

[Sh1] S. Shelah, Stability, the finite cover property and superstability, Ann. Math. Log (1971), 3, 271-362.

[Sh2] S. Shelah, Categoricity of uncontable theories, in Proc. of the Tarski Symposium, Proc. Symposium Pure Math XXV (1971), Univ. of Calif. Berkeley, 187-203.

[Va1] R. Vaught, Denumerable models of complete theories, Infinitistic Methods, Pergamon Press, 1961, 303-321. 


\section{Notation Index}

$\begin{array}{ll}\alpha, \beta, \gamma, \delta, \ldots & 5 \\ \kappa, \rho, \mu, \ldots & 5 \\ \omega, \omega_{1}, \omega_{2}, \ldots & 5 \\ \operatorname{card} A & 5 \\ \kappa^{+} & 5 \\ \lambda & 5 \\ \text { iff } & 6 \\ \square & 6 \\ \tau & 7 \\ I, J, K & 7 \\ R_{i}^{\mathcal{A}} & 7 \\ f_{j}^{\mathcal{A}} & 7 \\ c_{k}^{\mathcal{A}} & 7 \\ \mathcal{A}, \mathcal{B}, \mathcal{C}, \ldots & 7 \\ A, B, C, \ldots & 7 \\ \mathcal{A} \rightarrow \mathcal{B} & 9 \\ i_{A} & 9 \\ \mathcal{A} \subset \mathcal{B} & 9 \\ \mathcal{A} \approx \mathcal{B} & 9 \\ \mathcal{L}_{\tau} & 11 \\ R_{i} & 11 \\ f_{j} & 11 \\ \underline{c}_{k} & 11 \\ \sim & 12 \\ \& & \end{array}$




\begin{tabular}{|c|c|}
\hline$E$ & 12 \\
\hline$\left(E x_{i}\right)$ & 12 \\
\hline$\vee, \rightarrow, \leftrightarrow$ & 12 \\
\hline$\left(x_{i}\right)$ & 12 \\
\hline$\models$ & 14 \\
\hline$\equiv$ & 15 \\
\hline$\stackrel{\equiv}{\longrightarrow}$ & 16 \\
\hline$\prec$ & 17 \\
\hline$\vdash$ & 18 \\
\hline$T_{1}=T_{2}$ & 21 \\
\hline$T \mathcal{A}$ & 21 \\
\hline$D \mathcal{A}$ & 24 \\
\hline $\lim$ & 32 \\
\hline $\overrightarrow{T^{\mathcal{S}}}$ & 37 \\
\hline $\mathcal{A}^{\mathcal{S}}$ & 38 \\
\hline $\mathcal{A}^{\mathcal{S}}(x)$ & 38 \\
\hline$S_{n} T$ & 49 \\
\hline$\beta(c)$ & 50 \\
\hline $\mathrm{ACF}_{0}$ & 28,70 \\
\hline$n(T)$ & 77 \\
\hline$\langle\kappa, \rho\rangle$ & 82 \\
\hline$R^{\mathcal{A}}$ & 82 \\
\hline $\mathcal{L}_{\tau}^{\omega}$ & 93 \\
\hline $\mathcal{A}^{\omega}$ & 93 \\
\hline $\mathcal{K}$ & 101 \\
\hline $1_{\mathcal{A}}$ & 101 \\
\hline $\lim _{\leftarrow} \mathcal{A}_{i}$ & 103 \\
\hline $\mathcal{H}$ & 104 \\
\hline$F: \mathcal{K}_{1} \rightarrow \mathcal{K}_{2}$ & 103 \\
\hline$F f$ & 103 \\
\hline$F \mathcal{A}$ & 103 \\
\hline$f^{-1} x, f^{-1}[U]$ & 104 \\
\hline$\kappa(T)$ & 106 \\
\hline
\end{tabular}




\begin{tabular}{|c|c|c|}
\hline & $\mathcal{A}(b)$ & 106 \\
\hline & $S \mathcal{A}$ & 106 \\
\hline & $S f$ & 107 \\
\hline & $d X$ & 109 \\
\hline & $a_{X}$ & 109 \\
\hline$\frac{0}{\underline{e}}$ & $D F \mathcal{A}$ & 112 \\
\hline $\begin{array}{l}\tilde{w} \\
5 \\
0\end{array}$ & $D$ & 112 \\
\hline 这 & $D F f$ & 112 \\
\hline ర్๊ & $D^{\alpha} F$ & 113 \\
\hline 草 & $\operatorname{rank} x$ & 114 \\
\hline 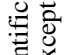 & $\operatorname{deg} x$ & 115 \\
\hline 列 & $f^{D E}$ & 118 \\
\hline & $D^{\alpha} S$ & 121 \\
\hline 管 & $\mathcal{V}(Y)$ & 122 \\
\hline 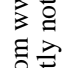 & $\mathcal{V}\left(y_{1}, \ldots, y_{n}\right)$ & 122 \\
\hline $8 . \bar{E}$ & $\alpha_{T}$ & 124 \\
\hline 焉. & $d_{T}$ & 125 \\
\hline 产 & $T^{\mathcal{M}}$ & 161 \\
\hline 焉 & $\emptyset$ & 164 \\
\hline 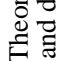 & $S \emptyset$ & 164 \\
\hline$\frac{\bar{g}}{2}$ & $T \cup q$ & 164 \\
\hline$\sigma^{\circ}$ & $p$-dim & 166 \\
\hline 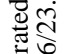 & $\operatorname{dim} \mathcal{A}$ & 183 \\
\hline 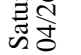 & $D x, D F_{0}$ & 185 \\
\hline $\begin{array}{c}\tilde{0} \\
n\end{array}$ & ord $f(x)$ & 186 \\
\hline in & $\mathrm{DCF}_{0}$ & 187 \\
\hline $\overrightarrow{\tilde{d}}$ & $\underline{\mathrm{DCF}_{p}}$ & 190 \\
\hline$\frac{\infty}{3}$ & $\overline{\mathcal{A}}$ & 193 \\
\hline
\end{tabular}


This page intentionally left blank 


\section{Index}

A-rank, 153

$R$-saturated, 88

$Z$-group, 63

$\kappa$-categorical, 2, 66

$\kappa$-dense, 51,52

$\kappa$-saturated, 51

$\kappa$-stable, 69

$\omega$-consistent, 94

$\omega$-logic, 93, 94

$\omega$-stable, 69

$\omega$-structure, 93

$n$-place function, 7

$n$-place relation, 7

$n$-type, 48

$p(\bmod q)$-dimension, 174

$p$-base, 166

$p$-dimension, 166

$p\left(\bmod \left\langle\mathcal{A}, a_{1}, \ldots, a_{n}\right\rangle\right)$-dimension, 174

absoluteness, 1, 54, 126, 196-198

admits direct limits, 102

admits filtrations, 101

admits filtrations with amalgamation, 101

admits inverse limits, 103

algebraic over $\mathcal{A}, 134$

algebraic over $X, 165$

algebraically closed, 28

algebraically independent, 165

antisymmetric over, 144, 145

atom, 64

atomic extension, 129

atomic formula, 11

atomic model, 78

atomic over, 129

autonomous, 117
Cantor-Bendixson derivative, 109

Cantor-Bendixson rank, 109

cardinality, 1, 162

cardinality of $\mathcal{A}, 8$

category, 101

complete, 21

connected over, 144, 145

consequence relation, 18

consistent, 18

constant term, 11

contravariant functor, 103

countability proviso, 47

countable direct system, 118

definable relation, 145

degree, 115

degree of Morley sequence, 146

diagram completed as shown, 5, 6

diagram of $\mathcal{A}, 24$

differential algebraic, 186

differential closure, 193

differential transcendental, 186

differentially closed field, 187

dimension of $\mathcal{A}, 164$

direct limit, 32-34, 102

direct system, 32-34, 102

directed set, 32, 102

distinguished element, 7, 33

downward Skolem-Löwenheim, 38

elementarily equivalent, 15

elementary extension, 17

elementary monomorphisms, 16

elementary partial automorphism, 72

elementary substructure, 17 
elimination of quantifiers, 37

endomorphism, 9

finitary character, 18

finite basis property, 126

finitely generated, 56

finitely generated extension of $T, 164$, 165

finitely generated over $\mathcal{V}, 122$

first order language, 11

formula, 11, 12

free variable, 12

full subcategory, 103

function, 7

function symbol, 11

generic solution, 186

homogeneous, 72

immediately extendible, 72

inclusion map, 121

indiscernible, 143

indiscernible over, 145

individual constant, 11

inverse limit, 2, 102-105

inverse system, 102-105

irreducible, 187, 193, 194

isolated point, 64, 105

isomorphic over $\mathcal{A}, 23,106$

isomorphism, 9, 15

limit ordinal, 5

limit point, 109

map, 9, 73

minimal generator, 164

minimal model, 80, 162

minimal model extension, 133, 162

minimal over, 133

model complete, 24

model completion, 39

monomorphism, 9

Morley derivative, 2, 101, 104, 109, 112
Morley prime model extension, 131, 134

Morley rank of $T, 124,196$

Morley sequence, 146-148

morleyization, 161

naming relation, 89-91

nonprincipal minimal generator,

174-177

normal extension, 132, 157

normal over, 132, 133

object, 10, 101-103, 156, 164, 168

omit an $n$-type, 64

order indiscernible, 138

order indiscernible over, 145, 146

ordinal, 5

ordinal recursive in $T, 1,126$

preserves limits, 103

prime model, $78-81$

prime model extension, 127-131

prime, proper elementary extension,

167,168

primitive symbol, 11

principal $n$-type, 129,130

quantifierless formula, 43, 46

quasi-totally transcendental, 125, 128

rank (Morley), 2, 3, 114

rank of $c$ in $\mathcal{D}, 179$

real closed field, 16, 29, 40, 58, 61, 62

realize, $2,4,15$

realize $p \in S_{n} T, 49,67,84$

realize the same $n$-types, 75

relation, 7

relation symbol, 11

restriction of $H$ to $K, 83$

satisfy, 15

saturated, 51

sentence, 12

similarity type, 9

simple extension, 59

singular cardinal, 5 
Skolem hull, 38

Skolemization, 37

special model, 71, 151

splits, 121

structure, $2,4,7$

subcategory, 103

substructure, 9

substructure complete, 43

substructure proviso, 145

successor ordinal, 5

term, 11

theory, 21

totally transcendental theory, 116, 125

two-cardinal type, 82,91

underlie $\mathcal{A}, 13$

universal closure, 15, 20 universal domain, 121-124

universal existential theory, 29, 187

universal formula, 29, 30

universal property of direct limit, 102, 103

universal theory, 45, 63, 185, 197

universe, 7

upward Skolem-Löwenheim, 22

valid, 15,89

Vaughtian pair for $(T, F(x)), 177,178$

Vaughtian pair for $T, 85,177$

weakly saturated, $77,78,80$ 